\title{
Structural, chemical and isotopic examinations of interstellar organic matter extracted from meteorites and interstellar dust particles
}

\author{
Henner Busemann ${ }^{1}$, Conel M. O'D. Alexander ${ }^{2}$, Larry R. Nittler ${ }^{2}$, \\ Rhonda M. Stroud ${ }^{3}$, Tom J. Zega ${ }^{3}$, George D. Cody ${ }^{4}$, \\ Hikaru Yabuta ${ }^{4}$, and A.L. David Kilcoyne ${ }^{5}$ \\ ${ }^{1}$ Planetary and Space Sciences Research Institute, The Open University, U. K. \\ email: h.busemann@open.ac.uk \\ ${ }^{2}$ Department of Terrestrial Magnetism, Carnegie Institution of Washington \\ ${ }^{3}$ Materials Science and Technology Division, Naval Research Laboratory Washington \\ ${ }^{4}$ Geophysical Laboratory, Carnegie Institution of Washington \\ ${ }^{5}$ Chemical Science Division, Berkeley National Laboratory
}

\begin{abstract}
Meteorites and Interplanetary Dust Particles (IDPs) are supposed to originate from asteroids and comets, sampling the most primitive bodies in the Solar System. They contain abundant carbonaceous material. Some of this, mostly insoluble organic matter (IOM), likely originated in the protosolar molecular cloud, based on spectral properties and $\mathrm{H}$ and $\mathrm{N}$ isotope characteristics. Together with cometary material returned with the Stardust mission, these samples provide a benchmark for models aiming to understand organic chemistry in the interstellar medium, as well as for mechanisms that secured the survival of these fragile molecules during Solar System formation. The carrier molecules of the isotope anomalies are largely unknown, although amorphous carbonaceous spheres, so-called nanoglobules, have been identified as carriers. We are using Secondary Ion Mass Spectrometry to identify isotopically anomalous material in meteoritic IOM and IDPs at a 100-200 nm scale. Organics of most likely interstellar origin are then extracted with the Focused-Ion-Beam technique and prepared for synchrotron X-ray and Transmission Electron Microscopy. These experiments yield information on the character of the $\mathrm{H}$ - and N-bearing interstellar molecules: While the association of $\mathrm{H}$ and $\mathrm{N}$ isotope anomalies with nanoglobules could be confirmed, we have also identified amorphous, micron-sized monolithic grains. D-enrichments in meteoritic IOM appear not to be systematically associated with any specific functional groups, whereas ${ }^{15} \mathrm{~N}$-rich material can be related to imine and nitrile functionality. The large ${ }^{15} \mathrm{~N}$ - enrichments observed here $\left(\delta^{15} \mathrm{~N}>1000 \%\right)$ cannot be reconciled with models using interstellar ammonia ice reactions, and hence, provide new constraints for understanding the chemistry in cold interstellar clouds.
\end{abstract}

\section{Discussion}

IRVINE: What is a 'nano-globule'?

BUSEMANN: It's a hollow piece of amorphous carbon that has a roundish shape, which is why it's called a 'globule.' We see this shape in the TEM and these show isotopic anomalies.

Bernstein: Could you be more specific about the correlation between the ${ }^{15} \mathrm{~N}$ isotopic anomalies and the functional groups? In your abstract you mention that the isotopic 
anomalies are associated in imines and nitriles. However, in one of your slides you showed it associated with amorphous carbon and talked about the ${ }^{15} \mathrm{~N}$ being associated with $\mathrm{NH}$, amino, and then in another slide it was nitrile and amide.

Busemann: The regions examined with the different techniques are not the same. The infrared information is from an enriched region shows only $\mathrm{OH}$ and $\mathrm{NH}$. If you look at the XANES spectra you see that the imine and the nitrile are both definitely enhanced. I see the most pronounced enrichment in the nitrile, but the imine group is very close to it in frequency space, so it is likely that there is some contribution from that as well.

Mumma: Could you clarify the changes in the Charnley and Rogers model that now produces predictions of enriched ${ }^{15} \mathrm{~N}$ even larger than the values you observed?

BusEmAnN: I don't understand it fully, but I think they freeze CO out of the gas phase relative to the nitrogen and this results in higher ${ }^{15} \mathrm{~N}$ enrichments in the gas phase chemistry.

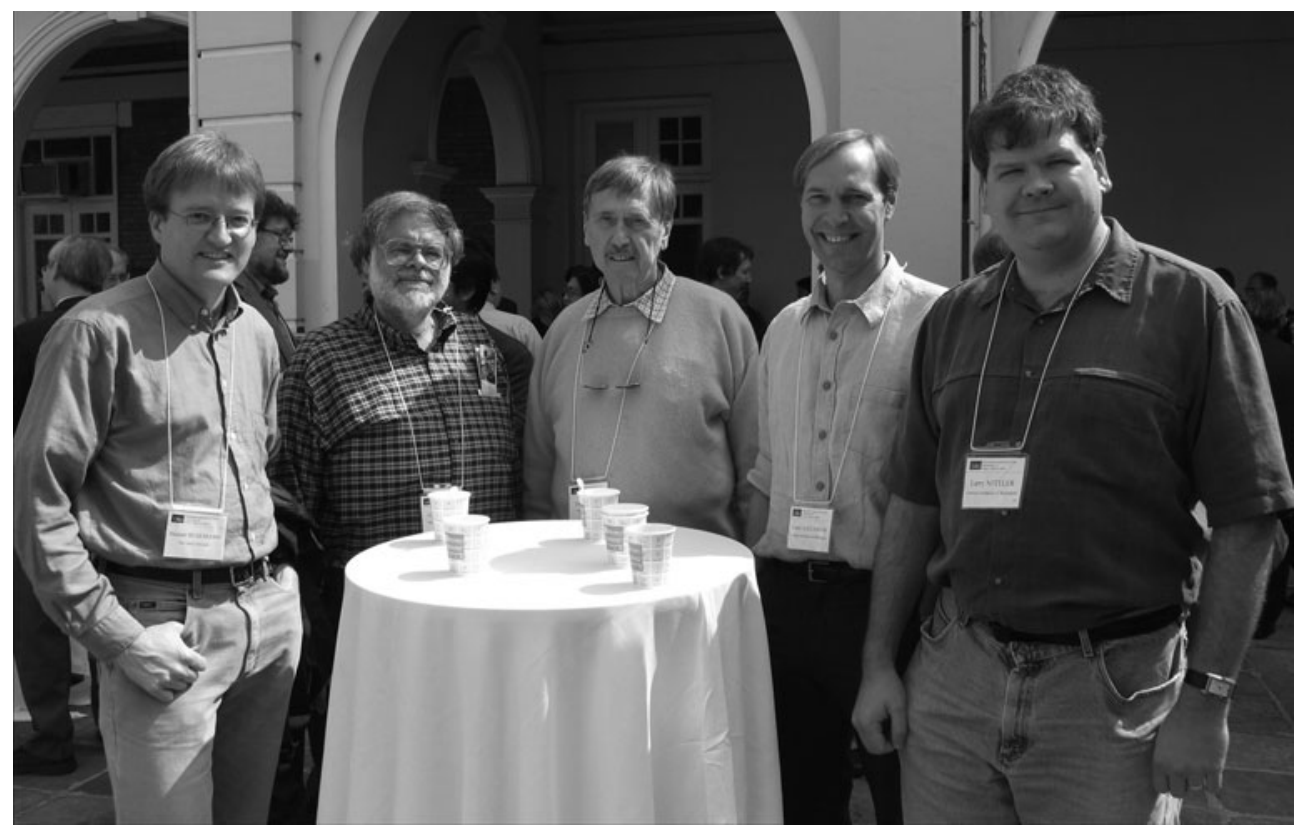

From left to right: Henner Busemann, George Flynn, Ernst Zinner, Conel Alexander, and Larry Nittler (photo by Dale Cruikshank). 